\title{
Bioequivalence Study of Two Formulations That Contain Isotretinoin 20 mg Capsules in Healthy Colombian Volunteers
}

\author{
Vargas $\mathbf{M}^{1 *}$, Villarraga $\mathrm{E}^{1}$, Batista $\mathbf{M}^{2}$, Montenegro $\mathrm{L}^{3}$ and Mantilla $\mathbf{P}^{3}$ \\ ${ }^{1}$ Pharmacology Unit, Universidad de la Sabana, Bogotá, Colombia \\ ${ }^{2}$ Pharmaceutical Chemistry Support, Procaps, Colombia \\ ${ }^{3}$ Medical Department, Procaps, Colombia
}

\begin{abstract}
This is a pharmacokinetic test of two formulations that contain $20 \mathrm{mg}$ of Isotretinoin, with the aim to compare the bioavailability between the Test Product (Isoface ${ }^{\circledR}$ from Procaps Laboratory SA, Colombia) and the Reference Product (Roaccutane ${ }^{\circledast}$ from Catalent Laboratory Germany, Eberbach GMBH, Germany), to declare the bioequivalence between both formulations. An open, crossed study was developed, randomized, of two periods and two sequences, with $40 \mathrm{mg}$ single dose of isotretinoin, on fasting conditions, on 24 healthy male Colombian volunteers; the washout was 14 days in between each period. To present the results, curves of the plasma concentration ratio vs. Time until hour 72 were performed; with the aim to identify the concentration provided by the Test formulation, the basal status of each volunteer was eliminated from the analysis, which was built with 3 former concentrations to the test medicine administration. The analytical method used in this study was high resolution liquid chromatography with tandem mass spectrometry detector, HPLC MS/MS, for plasma Isotretinoin identification and quantification. The main pharmacokinetic parameters for the Test Product vs. the Reference Product were $\mathrm{T}_{\text {máx }} 2.6 \mathrm{vs} .2 .8 \mathrm{~h}, \mathrm{C}_{\text {máx }} 190.5$ vs. $186.5 \mathrm{ng} / \mathrm{mL}$ for the $\mathrm{AUC}_{0-\mathrm{t}} 3003.8$ vs. $2933.5 \mathrm{~h} n \mathrm{ng} / \mathrm{mL}$ and $A U C_{0-\infty} 3726.3 \mathrm{vs} .3521 .2 \mathrm{~h}$ "ng $/ \mathrm{mL}$.

The confidence interval calculation of data with logarithmic transformation, showed confidence intervals for the variables $T_{\text {max }}, C_{\text {max }}, A \cup C_{0-t}$ and $A U C_{0-\infty}$, with values between 80-125; values approved by the FDA and EMA European Medicines Agency, in their Bioavailability and Bioequivalence Guides, to accept the Bioequivalence hypothesis between the two formulations in study, and thereby declare Bioequivalence and interchange ability of the Test Product from Procaps Laboratory, with the Reference Product from Roche Laboratory.
\end{abstract}

Keywords: Bioequivalence; Isotretinoin; Acne; Bioavailability; Pharmacokinetic

\section{Introduction}

Retinoids are intracrine and paracrine mediators of the cell differentiation, intervening in the reproduction, proliferation and apoptosis, by binding to the nuclear retinoic receptors. There are two types of retinoic receptors, the RXRs (x retinoid receptors) and the RARs (retinoic acid receptors). Each one of them, is as well divided into $\mathrm{a}, \mathrm{b}$ and $\mathrm{g}$. All these receptors are structurally alike, but show different affinities towards the different retinoid types, and, their body distribution differs, which explains the multiplicity of physiological processes they intervene [1].

Isotretinoin acts preferably on RAR receptors. Its effects on acne and other dermatological serious conditions are due to its action on the four pathogenetic factors:

- Sebum suppression Isotretinoin is an effective agent in the reduction of sebaceous glands size (more than 90\%).

- Inhibition of intraductal hyperkeratinisation Isotretinoin inhibits the proliferation of follicular keratinocytes and alters their terminal differentiation towards non-keratinized epithelia.

- Inhibition of Propionibacterium acnes growth. It has indirect bactericidal effect against $P$. acnes; its inhibitory effect over sebum production allows it to act on the follicular microenvironment.

- Anti-inflammatory properties Isotretinoin is a powerful B4 leukotriene and collagenase inhibitor, which induce polymorphonuclear cell migration on skin [1].
Isotretinoin is absorbed in the gastrointestinal tract, being the interindividual variation considerable in bioavailability. After the 40 $\mathrm{mg}$ oral administration of Isotretinoin to fasting healthy volunteers, the maximum plasma concentrations reported ranged from 167-459 ng/ $\mathrm{mL}$, reached in $3.2 \mathrm{~h} \mathrm{[2]}$; same dose in acne patients caused maximum plasma concentrations ranging from $98-535 \mathrm{ng} / \mathrm{mL}$ (average $262 \mathrm{ng} /$ $\mathrm{mL}$ ) in 2-9 h average [3]. Isotretinoin bioavailability increases from $1 \frac{1}{2}$ to 2 times more, when ingested with food. One of the main metabolites, 4-oxo-isotritenoine, is present in maximum plasma concentrations after $6 \mathrm{~h}$ of a single dose, remaining constant after 7 days Isotretionion's elimination half-life is $7-37 \mathrm{~h}$. The medicine binds to plasma proteins, almost exclusively to albumin (99.9\%). Another characteristic of this drug is that it crosses the placental barrier $[2,3]$. The elimination halflife on healthy subjects after a $40 \mathrm{mg}$ dose ranges from 10-20 h, being up to $90 \mathrm{~h}$ on patients who reach stationary states.

The main Isotretinoin metabolites detected in blood and urine are 4-oxo-isotretinoin and 4-hydroxi-isotretinoin, while other glucuronide conjugates are detected in bile. The 4-oxo-isotretinoin half-life is 29

*Corresponding author: Vargas M, M.Sc., DDS, Pharmacologist, Scientific Director Pharmacology Unit, Universidad de la Sabana, Bogotá, Colombia, Tel: + 578615555 ; E-mail: mauricio.vargas@unisabana.edu.co

Received October 22, 2016; Accepted October 31, 2016; Published November 11, 2016

Citation: Vargas M, Villarraga E, Batista M, Montenegro L, Mantilla P (2016) Bioequivalence Study of Two Formulations That Contain Isotretinoin $20 \mathrm{mg}$ Capsules in Healthy Colombian Volunteers. J Bioequiv Availab 8: 274-277. doi: 10.4172/jbb.1000308

Copyright: ( 2016 Vargas M, et al. This is an open-access article distributed under the terms of the Creative Commons Attribution License, which permits unrestricted use, distribution, and reproduction in any medium, provided the original author and source are credited. 
$\mathrm{h}(11-50 \mathrm{~h})$, being its metabolism performed predominantly in the liver. The 4-oxo-isotretinoin seric concentration is usually higher to isotretinoin after $6 \mathrm{~h} \mathrm{[2].}$

Isotretinoin's transportation in plasma occurs through union with albumin. Epidermic concentration is very low, and has not been found a progressive accumulation in serum, epidermic or subcutaneous cell tissue. Once treatment is discontinued, Isotretinoin disappears from serum and skin in 2-4 weeks [2,3]. According to FDA and EMA, two products that contain the same active principle are bioequivalent if they meet the conditions above $[4,5]$.

a) Calculate $\mathrm{ABC}$ (of test product)/ $\mathrm{ABC}$ (of reference product) for each test subject. The average and confidence interval of $90 \%$ of this quotient should be in a range of bioequivalence between 0.80 to 1.25 and corresponding percentage from $80 \%$ to $125 \%$ (for logarithmically transformed data). When data are analysed without transformation, the equivalence range is between 0.80 and $1.20(80 \%$ to $120 \%)$

b) Calculate $\mathrm{C}_{\max }$ (of test product) $/ \mathrm{C}_{\max }$ (of reference product) for each test subject. The average and confidence interval of $90 \%$ of this quotient should be in a range of bioequivalence between 0.80 to 1.25 or corresponding percentage from $80 \%$ to $125 \%$ (for logarithmically transformed data). When data are analysed without transformation, the equivalence range is between 0.80 and $1.20(80 \%$ to $120 \%)$.

c) Calculation of any other variable to declare two products bioequivalents must be fully justified.

This report corresponds to the biopharmaceutical results in 24 healthy, male, Colombian, volunteers with a single dose regimen of $40 \mathrm{mg}$ strength, tending to evaluate bioavailability of Isotretinoin formulation between the Test Product Isoface from Procaps SA (Colombia) and Reference Product Roaccutane from Catalent Germany, Eberbach GMBH (Germany).

\section{Study Design}

It was used an open, randomized, two periods, two sequences, crossed and 14 days washout in between periods design. Three days before the beginning of each period, the volunteers had to abstain from medicines, alcohol and any food or beverage containing methylxanthines. These restrictions were maintained throughout the time that the samples were obtained. All volunteers were randomized to be assigned to the treatment sequence.

\section{Materials and Methods}

\section{Formulations in study}

Test medicament: Isotretinoin, Isoface for oral administration that contains Isotretinoin $20 \mathrm{mg}$, manufactured and distributed by Procaps Laboratories SA, Lot 1072274 Expires April 2018.

Reference medicament: Isotretinoin, Roaccutane for oral administration that contains Isotretinoin $20 \mathrm{mg}$, manufactured and distributed by Catalent Germany, Eberbach GMBH, Germany, and Lot B9254B03 Expires 04/2018.

Subjects: Before the clinical phase, the volunteers went under medical examination and laboratory tests in order to confirm their health status. Histories of alcoholism, diseases with compromised liver or kidney function, blood dyscrasias or proteinuria were the considered exclusion factors.
Medical examination and laboratory tests: The requested clinical laboratory tests were complete blood count, total and direct bilirubin, creatinine, glucose, total protein, and complete urinalysis, Elisa for $\mathrm{HIV}$, antibodies to hepatitis $\mathrm{C}$ and $\mathrm{B}$, and electrocardiogram.

\section{Obtaining informed consent}

The Protocol and Informed Consent were authorized by the Ethics Committee in Clinical Investigation (CEIC) of Clínica de La Universidad de La Sabana, which is governed by the legal and ethical guidelines in Resolutions 008430 of 1993 and 002378 of 2008 of the Ministerio de la Protección Social de Colombia [6], the World Conference on Harmonization for good clinical practices in institutions that conduct research in humans, and by the principles of World Medical Assembly published in the Declaration of Helsinki, last reviewed in 2013 [7].

The 24 selected volunteers met the inclusion criteria summarized above This group of volunteers presented average age 31 years (range 20 - 48 years), average weight $71 \mathrm{~kg}(55-87 \mathrm{~kg})$, average size $173 \mathrm{~cm}(164-$ $183 \mathrm{~cm})$ and average IMC $23.7 \mathrm{~kg} / \mathrm{m}^{2}\left(19.0-28.7 \mathrm{~kg} / \mathrm{m}^{2}\right)$. All volunteers signed informed consent.

\section{Drug administration}

For the drug administration, the volunteers kept fasting for $10 \mathrm{~h}$. A $40 \mathrm{mg}$ [8] dose was ingested with $200 \mathrm{~mL}$ of water, meaning $220 \mathrm{mg}$ capsules to each volunteer; and $4 \mathrm{~h}$ later, each volunteer was provided with standardized food. During the stay period in the clinic, two meals (breakfast and lunch) and two snacks (one in the morning, one in the afternoon) were provided.

The team for obtaining the samples consisted of a doctor and a licensed nurse. All volunteers received either the Test or the Reference product by randomization. 14 venous blood samples were taken at the following times $-1,0,-2,0 \mathrm{~h}$ before drug administration, and 1 , $2,2.5,3,3.5,4,6,12,24,48$ years, $72 \mathrm{~h}$ after samples were labelled for identification and centrifuged at $4000 \mathrm{rpm}$ during 25 mints. Plasma was transferred into pre-labelled test cryovial and frozen at $-20^{\circ} \mathrm{C}$ for further analysis.

After 14 days of washout, the same procedure was repeated, crossing the administration and yet completing the second study period.

\section{Validation of the analytical method}

Validation was performed according to the bioanalytical methodology validation procedure established by QUASFAR M\&F SA.

- Selectivity No interference was evident in the different analysed targets.

- Matrix Effect $\pm 15 \%$ of the nominal concentration.

- Calibration proportionality between the response and the level of concentration is evident.

- The lowest curve point does not deviate in $\pm 20 \%$ and the other levels do not deviate in $\pm 15 \%$.

- Accuracy $\pm 15 \%$ of the nominal concentration.

- Precision $\pm 15 \%$ of the nominal concentration.

Calibration: Calibration was performed over enriched samples at levels 10,50,100,150, 300 years, $400 \mathrm{ng} / \mathrm{mL}$ Applying analytical method, 5 calibration curves were run at different days linear regression was performed to each curve.

Analytical conditions: Quantification limit was $1 \mathrm{ng} / \mathrm{mL}[3,9,10]$. 
Citation: Vargas M, Villarraga E, Batista M, Montenegro L, Mantilla P (2016) Bioequivalence Study of Two Formulations That Contain Isotretinoin 20 mg Capsules in Healthy Colombian Volunteers. J Bioequiv Availab 8: 274-277. doi: 10.4172/jbb.1000308

\section{Pharmacokinetic analysis}

The Pharmacokinetic Analysis was performed by WinNonlin 5.3 program (Pharsight Corporation, Cary USA), adjusted to a noncompartmental analysis. The maximum concentration $\left(\mathrm{C}_{\text {max }}\right)$ and the time to reach it $\left(\mathrm{t}_{\max }\right)$ were directly obtained from the serum concentrations results, as currently recommended by the FDA [4] and the EMA (European Medicines Agency) [5]. UC total was calculated by the sum of the partial AUC.

a) $\mathrm{AUC}_{0-\mathrm{t}}$, between time zero and the last time with detectable concentrations, calculated by the trapezoidal rule, and ensuring the calculation of at least the $80 \%$ of the AUC with the last sample.

b) $\mathrm{AUC}_{\mathrm{t}-\infty}$, calculated as the ratio $\mathrm{C} / \mathrm{K}$, being $\mathrm{C}$ the last detectable concentration and $\mathrm{K}$ the slope of the line, obtained by linear regression, from the points corresponding to the elimination phase of the drug, by linear regression of the natural logarithm of the concentrations [11]. The elimination rate constant $\left(\mathrm{K}_{\mathrm{e}}\right)$, half-life $\left(\mathrm{t}_{1 / 2}\right)$, the clearance $(\mathrm{Cl})$ and the mean residence time (MRT), adjusted to Bioavailability, were calculated after the non-compartmental analysis.

\section{Statistical analysis}

Variance Analysis (ANOVA) was used to determine possible effects for each variation factor, per sequence, period or subjects. The F-test was used with a statistical significance level of $5 \%(\alpha=0$, $0.5 \%)$. The statistical comparison of the transformed pharmacokinetic parameters on both formulations was performed using the statistical program WinNonlin version 5.3.

The following bioequivalence criteria were established on the protocol the Confidence Interval of $90 \%$ of $\mathrm{C}_{\text {máx }}$ test/ $\mathrm{C}_{\text {máx }}$ Reference and last test $\mathrm{AUC} /$ last reference AUC, relations must be in the range of $80-125 \%$ of acceptability Plus, $A C_{t}$ must not be less than $80 \%$ of AUC total [11,12].

\section{Results}

24 healthy male volunteers with Colombian nationality completed the two periods and were included in the pharmacokinetic and statistical analyses (Graph 1).

Table 1 shows the pharmacokinetic parameter averages obtained from all volunteers (average \pm SD), with both studied formulations. For the pharmacokinetic analysis, the three baseline points were taken $(-10$, -2 years, $0 \mathrm{~h}$ ) and an average value was obtained. This average value was eliminated from the obtained individual values for each volunteer and for each formulation, and thus we obtained the plasma concentrations provided by the studied formulations.

Table 2 shows confidence intervals of $90 \%$, of the logarithmically transformed pharmacokinetic parameters, analyzes to determine if bioequivalence exists between Test Product, Isoface of Procaps SA, and Reference Product, Roaccutane of Catalent Germany, Eberbach GMBH, Germany.

\section{Discussion}

Health resources control is a need for all nations. Bioequivalence studies are the surrogate proof that makes us think with a high degree of probability, that the two generic drugs will have a similar efficacy and safety profile, i.e., are therapeutic equivalents [12].

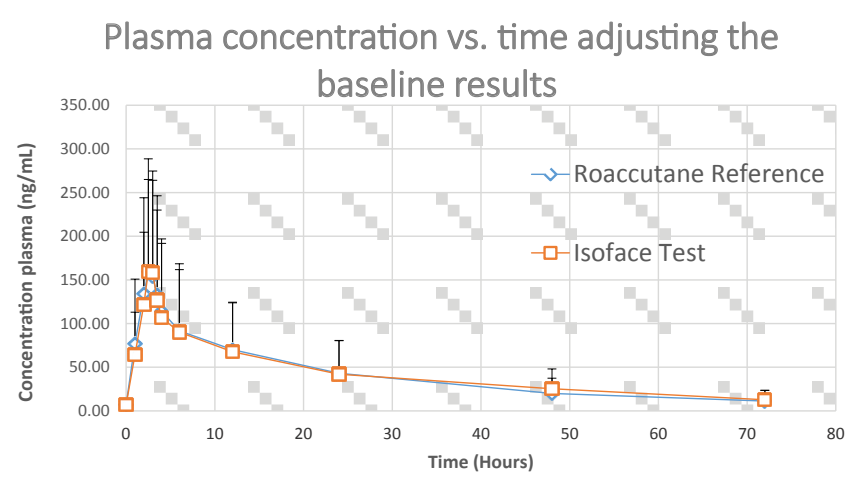

Graph 1: Bioavailability curve (Concentration vs. Time) obtained after a single 40 $\mathrm{mg}$ isotretoine dose administration in fasting conditions of test product (Isoface ${ }^{\circledR}$ from Procaps SA, Colombia) and reference product (Roaccutane ${ }^{\circledR}$ from Catalent Germany, Eberbach GMBH, Germany).

\begin{tabular}{|c|c|c|c|c|c|c|}
\hline Treatment & \begin{tabular}{|c|} 
Elimination \\
Rate $(1 / \mathrm{h})$
\end{tabular} & $\begin{array}{l}\text { Half-life } \\
\text { (h) }\end{array}$ & $t_{\text {máx }}(h)$ & $\mathrm{C}_{\text {máx }}(\mathbf{n g} /$ & $\begin{array}{c}A^{A U C} C_{0-t} \\
\left(h^{*} n g / m L\right)\end{array}$ & $\begin{array}{c}A_{\left(h^{*} n g / m L\right.} C_{\text {-Inf }} \\
\end{array}$ \\
\hline Reference & 0.043 & 25.0 & 2.8 & 186.5 & 2933.5 & 3521.2 \\
\hline Test & 0.033 & 31.3 & 2.6 & 190.5 & 3003.8 & 3726.3 \\
\hline
\end{tabular}

Isotretinoin's pharmacokinetic parameters of test product (Procaps SA) and reference product (Roaccutane ${ }^{\circledR}$ ) following a $40 \mathrm{mg}$ single dose in fasting conditions

Table 1: Summarized pharmacokinetic parameter data (Adjusted to Baseline).

\begin{tabular}{|c|c|c|c|c|}
\hline \multicolumn{2}{|c|}{} & \multicolumn{2}{c|}{$\begin{array}{c}\text { Cl standard 90\% (Test/ } \\
\text { Reference) }\end{array}$} \\
\hline Parameter & Units & Ratio \% & $\mathbf{8 0}$ & 125 \\
\hline $\mathrm{Ln}\left(\mathrm{T}_{\max }\right)$ & $\mathrm{h}$ & 97.89 & 83.63 & 114.59 \\
\hline $\mathrm{Ln}\left(\mathrm{C}_{\text {máx }}\right)$ & $\mathrm{ng} / \mathrm{mL}$ & 101.01 & 88.25 & 115.62 \\
\hline $\mathrm{Ln}\left(\mathrm{AUC}_{\text {all }}\right)$ & $\mathrm{h}^{*} \mathrm{ng} / \mathrm{mL}$ & 95.76 & 80.86 & 113.40 \\
\hline $\mathrm{Ln}\left(\mathrm{AUC} \mathrm{C}_{\text {INF_obs }}\right)$ & $\mathrm{h}^{*} \mathrm{ng} / \mathrm{mL}$ & 101.95 & 84.77 & 122.61 \\
\hline
\end{tabular}

Table 2: Confidence intervals of $90 \%$ for isotretinoin formulations (Test and Reference Products) after administration to healthy volunteers.

Completion of the in vitro study, allows the comparison between the two studied formulations in order to declare the Pharmaceutical Equivalence, and qualify their quality attributes; in vivo studies allow declaring interchange ability of the active ingredients that do not meet the biowaiver [13].

The 24 initially included volunteers concluded the test, and no adverse events occurred with any of the two formulations studied in fasting conditions. Washout was greater than the 7-recommended elimination half-lives, and guaranteed the absence of traces between periods [14].

In the case of Isotretinoin, incorporation of a bioequivalent formulation in the pharmacological treatment for nodulocystic acne or with a serious scar component, contributes to the clinical aim and cost control of this disease.

\section{Conclusion}

Isotretinoin formulation manufactured by Procaps SA, Colombia, Isoface; Test Product, and formulation manufactured by Catalent Germany, Eberbach GMBH, Germany Roaccutane, Reference Product, have pharmacokinetic parameters that allow to declare bioequivalence between both formulations.

\section{References}

1. Feldman S, Careccia RE, Barham KL, Hancox J (2004) Diagnosis and Treatment of Acne. Am Fam Physician 69: 2123-1232. 
Citation: Vargas M, Villarraga E, Batista M, Montenegro L, Mantilla P (2016) Bioequivalence Study of Two Formulations That Contain Isotretinoin 20 mg Capsules in Healthy Colombian Volunteers. J Bioequiv Availab 8: 274-277. doi: 10.4172/jbb.1000308

2. Abo-Talib N, Tammam M, Hassan E (2012) Determination of isotretinoin in human plasma: Application to pharmacokinetic study. Bull Fac Pharmacy 50: $127-132$

3. Agarwal US, Besarwal RK, Bhola K (2011) Oral isotretinoin in different dose regimens for acne vulgaris: A randomized comparative trial. Indian J Dermatol Venereol Leprol 77: 688-694.

4. Food and Drugs Administration (2001) Guidance for industry: Statistical Approaches to Establishing Bioequivalence. U.S. Department of Health and Human Services.

5. The European Agency for the Evaluation of Medicinal Products (EMA) (2001) Committee for Proprietary Medicinal Products (CPMP): Note for Guidance on the Investigation of Bioavailability and Bioequivalence, London, UK.

6. Republic of Colombia Ministry of Health (1993) Why scientific and administrative rules, established techniques for health research. Resolution No. 008430.

7. World Medical Assembly (2013) Declaration of Helsinki of the AMM-Ethica Principles for Medical Research in humans, 64 ${ }^{\text {th }}$ General Assembly, Fortaleza, Brazil.
8. Jones K, O'Donovan D, Horowitz M, Russo A, Lei Y, et al. (2006) Effects of posture on gastric emptying, transpyloric flow, and hunger after a glucose drink in healthy humans. Dig Dis Sci 51: 1331-1338.

9. U.S. Department of Health and Human Services Food and Drug Administration Center for Drug Evaluation and Research (CDER) Center for Veterinary Medicine (CVM) (2013) Guidance for Industry: Bioanalytical Method Validation.

10. Committee for Medicinal Products for Human Use (CHMP), Europeam Medicines Agency (EMEA) (2009) Guidance on Validation of Bioanalytical Methods, London UK.

11. Perry R (2010) Perspectives on the bioequivalence and therapeutic equivalence of generic formulations: An overview of the landscape. Clin Ther Sep 32: 1796-1797.

12. World Health Organization (2005) Multisource (Generic) Pharmaceuticals Products Guidelines on Registration Requirements to Establish Interchangeability. WHO Technical Report Series.

13. World Health Organization (2006) Public Health: Innovation and Intellectua Property Rights. Commission on Intellectual Property Rights and Public Health pp: 1-188.

14. Julious SA (2004) Tutorial in biostatistics: Sample sizes for clinical trials with normal data. Stat Med 23: 1921-1986. 